\title{
Initial Brazilian Experience in the Treatment of Localized Prostate Cancer Using A New Generation Cryotechnology: Feasibility Study
}

Fernando J. Kim, Michael A. Cerqueira, Jose C. Almeida, Alexandre Pompeo, David Sehrt, Jose M. Calheiros, Fernando A. Martins, Wilson R. Molina

Denver Health Medical Center, Denver (FJK, MAC, AP, DS WRM), Colorado, USA, Hospital das Forças Armadas (HFA) (JCA, JMC), Brasília, ABC Medical School (AP), São Paulo and Hospital Beneficência Portuguesa (MAC, FAM), Rio de Janeiro, Brazil

\section{ABSTRACT}

Introduction: The objective of our study is to present the first Brazilian cryoablation experience in the treatment of low and intermediate risk localized prostate cancer using 3rd generation cryoablation and real-time biplanar transrectal ultrasonography.

Materials and Methods: Ten Brazilian patients underwent primary cryoablation for localized prostate cancer between October 2010 and June 2011. All patients consented for whole gland primary cryotherapy. The procedures were performed by 3rd generation cryoablation with the Cryocare System ${ }^{\circledR}$ (Endocare, Irvine, California). Preoperative data collection included patient demographics along with prostate gland size, Gleason score, serum prostate specific antigen, and erectile function status. Operative and post-operative assessment involved estimated blood loss, operative time, complications, serum PSA level, erectile function status, urinary incontinence, biochemical disease free survival (BDFS), and follow-up time.

Results: All patients in the study successfully underwent whole gland cryoablation. The mean of: age, prostate size, PSA level, and Gleason score, was 66.2 years old; 40.7g; $7.8 \mathrm{ng} / \mathrm{mL}$; and 6 respectively. All patients were classified as low or moderate D'Amico risk ( 5 low and 5 moderate). Erectile dysfunction was present in 50\% of patients. The estimated blood loss was minimal, operative time was 46.1 minutes. All patients that developed erectile dysfunction post-treatment responded to oral or intracavernosal medications with early penile rehabilitation. All patients maintained urinary continence by the end of a 10 months evaluation period and none had biochemical relapse within the mean follow-up of 13 months (7-15 months).

Conclusion: Our initial experience shows that cryoablation is a minimally invasive option for the treatment of localized prostate cancer. Short term data seems to be promising but longer follow-up is necessary to verify oncological and functional results.
ARTICLE INFO

\section{Key words:}

Cryoablation; Prostatic

Neoplasms; PSA

Int Braz J Urol. 2012; 38: 620-6

Submitted for publication: January 31,2012

Accepted after revision:

April 09, 2012

\section{INTRODUCTION}

Cryoablation is an accepted minimally invasive surgical option for the treatment of localized prostate cancer (1). It is estimated that in the
United States more than 6,500 cryoablation procedures were performed in 2005 , and $>15,000$ procedures in 2010 (2).

Currently, primary cryoablation is indicated to all patients that are eligible to other treatment 
modalities, including clinically localized disease in younger and low morbidity patients. Due to technical refinements such as improved ultrasonography imaging and routine use of urethral warmers and small gauge needle delivery systems, complication rates have significantly decreased and renewed the interest in this treatment modality (2-5).

Cryoablation triggers cell apoptosis with continuing cellular destructive mechanism and intracellular osmotic injury (6). The indirect mechanisms involve thrombosis, ischemia - reperfusion injury, and programmed cell death. Reports from the US and European academic centers demonstrated that cryosurgery for the treatment of prostate and renal cancer offers good oncological and functional outcomes and is gaining acceptance worldwide (7,8). In Brazil, the Agência Nacional de Vigilância Sanitária (ANVISA) approved the cryoablation technology in 2007 under the following registration codes: 80181930029 and 80181930032. The objective of our study is to present the first Brazilian cryoablation experience in the treatment of low and intermediate risk localized prostate cancer.

\section{MATERIALS AND METHODS}

A total of 10 male patients underwent primary cryoablation for localized prostate cancer between October 2010 and June 2011 and included in the study after institutional review board approval; an informed consent was obtained. All patients had biopsy-proven prostate cancer and were classified as low to intermediate risk according to D'Amico risk stratification. Preoperative data collection included patient demographic data, prostate gland size, Gleason score, serum prostate specific antigen (PSA), and potency.

Operative and postoperative assessment included estimated blood loss, operative time, complications, serum PSA, potency, urinary incontinence, biochemical disease free survival (BDFS), and clinical follow-up. Beginning at 3 months urinary incontinence and erectile dysfunction were assessed. Incontinence was defined as uncontrolled leakage of urine through the urethra requiring at least one male incontinence pad. Potency was diagnosed by a self-reported ability to achieve erection for in- tercourse without any pharmaceutical or vacuum device assistance. Biochemical disease free survival was determined using American Society of Therapeutic Radiation Oncology (ASTRO) criteria of three consecutive rises in PSA level and with the Phoenix criteria of PSA nadir plus $2 \mathrm{ng} / \mathrm{mL}$. Complications were graded using the Clavien-Dindo scale (9). Clavien I-II complications were considered minor while Clavien III-V were classified as major complication. Data collection and statistics used Microsoft Excel (Microsoft Corporation, Redmond, WA). Data were reported as mean \pm standard deviation and frequency (percentage of total).

All procedures were performed with Cryocare System $^{\circledR}$ (Endocare, Irvine, California). Cryoablation involved a dual freeze-active thaw cycles using 8-17 gauge cryoprobes with helium and argon gas. The patients were placed in a lithotomy position and cryoablation was performed under general anesthesia. Cryoprobes and thermocouples were placed with assistance of a brachytherapy grid guided by transrectal ultrasound (TRUS) in both the sagittal and the transverse planes. Thermoprobes were placed at the urinary sphincter and Denonvillier's fascia. The urinary sphincter and Denonvilliers thermocouples monitored temperatures close to $0^{\circ}$ $\mathrm{C}$ to protect the anterior rectal wall and urinary sphincter. Cystoscopy was conducted to confirm correct cryoprobes placement and that the needles did not penetrate into the urethra or bladder. A urethral warming catheter set at $42^{\circ} \mathrm{C}$ protected the urethra for the entirety of the procedure. A Foley catheter was placed following cryoablation. Patients were discharged within $24 \mathrm{hrs}$. post-cryoablation with the Foley catheter. On postoperative day 5 the catheter was removed. Patients returned for follow-up 1 month after the procedure, and every 3 months thereafter. Serum PSA was collected at every visit. Penile rehabilitation was performed in patients without preoperative erectile dysfunction. These patients were encouraged to use daily Phosphodiesterase 5 inhibitors (PDE 5 i), i.e., Tadalafil $5 \mathrm{mg}$ and a vacuum erectile device twice a day.

\section{RESULTS}

All patients in the study successfully underwent whole gland cryoablation as primary 
treatment for localized prostate adenocarcinoma. Table- 1 shows patient demographics and preoperative disease characteristics. Mean age was 66.2 years old. Average prostate size was 40.7 grams. Mean PSA was $7.8 \mathrm{ng} / \mathrm{dL}$ and mean Gleason Score was 6. Patients were evenly distributed between low and moderate according D'Amico Score. Half of the patients were defined as potent preoperatively. Mean follow-up for all patients was 13 months (7-15 months). Surgical outcomes are presented in Table-2. Blood loss was minimal in all procedures and operative time was $<60$ minutes. Figure- 1 depicts changes in PSA level; pre versus post-operative PSA for each patient and the average PSA level $(\mathrm{ng} / \mathrm{mL})$. Of note, the highest postoperative PSA $(1.44 \mathrm{ng} / \mathrm{mL}$ ) occurred in the patient with the largest prostate (70 grams) whose initial PSA was $5.51 \mathrm{ng} / \mathrm{mL}$. There was no biochemical relapse observed during this short-term clinical follow-up. All patients who underwent penile rehabilitation were able to achieve erection for penetration with pharmacological therapy. Two patients required PDE 5 inhibitors alone while the remaining three patients responded to intracavernosal therapy.

No intraoperative complications were observed and all patients were discharged within 24 hours post procedure. Table- 3 demonstrates postoperative complications. All complications were

Table 1 - Patient Demographic and Pre-operative Data.

\begin{tabular}{lc}
\hline Age $(\mathrm{yr})$ & $66.2 \pm 10.8$ \\
\hline Size $(\mathrm{g})$ & $40.7 \pm 16.4$ \\
PSA (ng/dL) & $7.8 \pm 2.8$ \\
Gleason & \\
6 & $6(60 \%)$ \\
7 & $4(40 \%)$ \\
D'Amico & \\
Low & $5(50.0 \%)$ \\
Moderate & $5(50.0 \%)$ \\
High & $0(0.0 \%)$ \\
Potency & $5(50.0 \%)$ \\
\hline
\end{tabular}

Table 2 - Surgical and Post-Operative Data.

\begin{tabular}{lc}
\hline EBL & Minimal \\
\hline ORT (min) & $46.1 \pm 3.3$ \\
Post-Operative PSA (mg/dL) & $0.54 \pm 0.64$ \\
Potency & $0(0.0 \%)$ \\
Unresponsive to Meds & $2(40.0 \%)$ \\
Responsive to Oral Meds & $5(100.0 \%)$ \\
Responsive to Oral or ICI Meds & $10(100.0 \%)$ \\
BDFS & $13.0 \pm 4.5$ \\
Follow-up Time $(\mathrm{m})$ & \\
\hline
\end{tabular}

$\mathbf{E B L}=$ Estimated Blood Loss; $\mathbf{O R T}=$ Operative Time; Meds = Medications; $\mathbf{C l}$ = Intracavernosal Injection; BDFS = Biochemical Disease Free Survival

Figure 1 - Changes in PSA; pre versus post-operative PSA for each patient and the average PSA level $(\mathrm{ng} / \mathrm{mL})$.

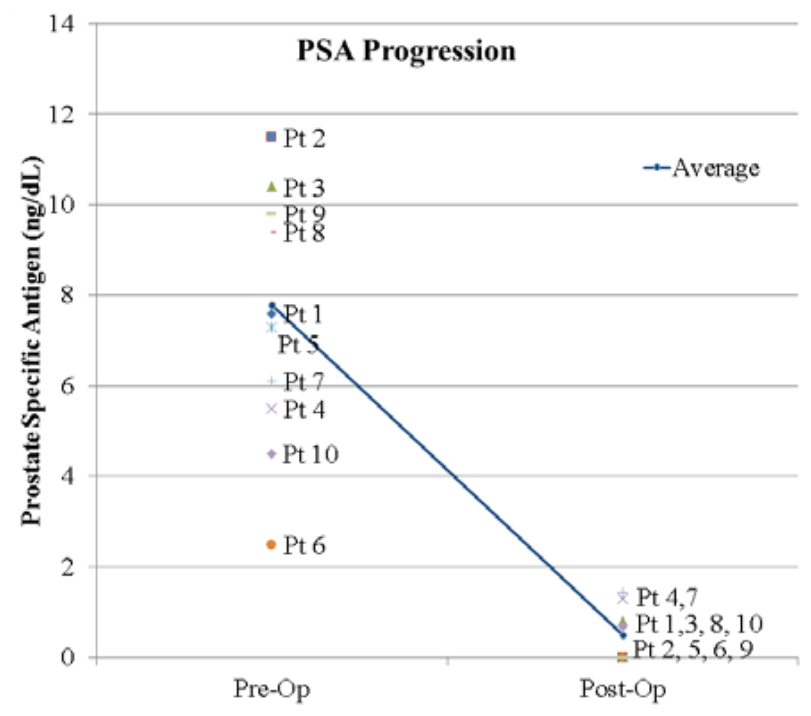

Clavien Grade I. Hematuria was observed in 3 patients. Two of the patients with hematuria were associated with coagulation issues, i.e., low platelet count and liver dysfunction. Complete resolution of hematuria occurred after medical therapy and clot evacuation. Two patients were diagnosed with urinary retention at 2 weeks postop and required Foley catheterization for an additional week. Both 
Table 3 - Complications.

\begin{tabular}{lcccc}
\hline Complications & Clavien Grade & Frequency & Management & Resolution \\
\hline Dysuria & I & $1(10.0 \%)$ & Observation & $6 \mathrm{~m}$ \\
Hematuria & $\mathrm{I}$ & $3(30.0 \%)$ & clot evacuation & 1 week \\
Perineal Pain & $\mathrm{I}$ & $1(10.0 \%)$ & Observation & - \\
Scrotal Hematoma & $\mathrm{I}$ & $1(10.0 \%)$ & scrotal support & 1 week \\
Urinary Retention & $\mathrm{I}$ & $2(20.0 \%)$ & Foley catheter & 1 week \\
\hline
\end{tabular}

patients voided without issues 3 weeks post-surgery. Scrotal hematoma was noted in one patient who was treated with a scrotal support and analgesic and resolved in 1 week. One patient had dysuria that was managed with reassurance and observation, and completely resolved at 6 months post-op.

\section{DISCUSSION}

Cryosurgery for prostate cancer was first applied in 1964 by Gonder et al. using liquid nitrogen (10). The technique encompassed transurethral freezing of the prostate without the proper technology to position the cryoprobes adequately and to monitor the extension of the ice ball. This resulted in severe and frequent complications such as urinary incontinence, urethral sloughing and recto-urethral fistulae. After 1980, Onik et al. refined the technique by using interventional radiological procedures and transrectal ultrasound (11). The TRUS - guided transperineal placement of cryoprobes with real - time monitoring and control of the freezing process has significantly decreased the complications $(12,13)$.

Since the use of urethral warmers, thermocouples in Denonvillier's fascia and neurovascular bundles and the application of gas - based cryosurgery, complication rates have further decreased $(14,15)$. The introduction of argon gas for freezing and helium gas for thawing permitted a dramatic reduction in the diameter of the cryoprobes. The ultrathin 17 - gauge $(1.47 \mathrm{~mm})$ cryoneedles have a very sharp tip and because of the smaller diameter, more needles can be placed. This permits a precise contouring of the ice ball, subsequently resulting in a more effective ablation of the gland (16). These developments have significantly minimized the scrotal swelling and perineal ecchymosis that occurred after the procedure (17). By active instead of passive thawing the procedure can be performed much quicker. Most patients are discharged from the hospital either the same day or the following day after treatment (7).

The use of a urethral - warming catheter decreased the sloughing rate of the urethral mucosa and subsequently the risk of obstructive problems $(2,5)$. Consequently, cryosurgery was recognized by the American Urological Association (AUA) as a therapeutic option for localized prostate cancer in 1996. Cryoablation is recognized as an established minimally invasive procedure for the treatment of localized prostate cancer in both the primary and salvage setting (18-20). The Brazilian Society of Urology initially determined cryoablation an experimental technique. The newest Brazilian recommendation published in 2011 ascertained the 2009 decision that cryoablation is a treatment option for localized prostate cancer, especially those with comorbidities (21).

While new advances in technology for the treatment of prostate cancer can be very costly with little clinical data to support large financial investment, i.e, robotic radical prostatectomy, cryotechnology may be a cost effective treatment modality, saving the institution and the patient significant resources (22). The evolution of cryoablation has further decreased the learning curve for procedure. The percutaneous needle ablation simplifies surgery compared to other therapies such as open, laparoscopic or robotic prostatectomy. 
Outcomes from the Cryo On-Line Data registry reported that among the 1.198 consecutive patients who underwent primary cryoablation, 3.6\% of patients developed urinary retention and $0.4 \%$ rates of recto-urethral fistulas (23). In our study, no recto-urethral fistula was observed. Two patients developed acute urinary retention that resolved after prolonged urethral catheterization.

Biochemical failure after cryoablation of prostate requires re-biopsy of the prostate. Other Brazilian investigators (Hayek et al.) published their recurrence rates with cryoablation in 21 high-risk patients in 2007 defining biochemical failure as PSA > $1.0 \mathrm{ng} / \mathrm{mL}$ using an older cryo system. The PSA failure rate was 39\%, 52.9\%, and $42.8 \%$ at 12,24 , and 60 months of followup, respectively. In that study 12 (57.2\%) patients had biochemical failure, while 7 (58.3\%) of these patients had positive prostate re-biopsy. They concluded that prostate cryoablation is a minimally invasive treatment with promising results, resulting in low complication rates for high risk patients with prostate cancer (24). Jones et al. using the COLD registry database reported a 5-year BDFS in 1.198 patients using the Phoenix criteria of $91 \%, 78 \%$, and $62 \%$ for low, moderate, and high risk respectively (23). Cohen et al. demonstrated a 10 year BDFS of $80.6 \%, 74.2 \%$, and $45.5 \%$ for low, moderate, and high risk patients using the Phoenix criteria (25). A randomized trial comparing radiation and cryoablation for patients with localized prostate cancer showed similar results regarding BDFS and overall survival after 3 years of follow-up between the two modalities (26). In our series, we demonstrate the first series of Brazilian patients with low and intermediate risk prostate cancer treated with cryoablative technology. Using ASTRO and Phoenix criteria, our short-term follow-up revealed no biochemical recurrence.

Reported incidence of urinary incontinence varies significantly due to lack of standard definition. Past studies have shown that the incidence of incontinence with modern cryotherapy may range from $1.3 \%$ to $7.5 \%$. Jones and Long reported in their series a total incontinence rate of $4.8 \%$ with $2.9 \%$ of patients requiring pads $(24,27)$. The 0\% urinary incontinence rate (no pads) in our present series may be due to a smaller group of patients and a biased population with low risk and healthier medical status. Two patients that presented with mild acute urinary incontinence post-surgery resolved spontaneously and/or with biofeedback therapy within 30 days post-op.

Historically, investigators believed that prostate cryoablation would permanently cause erectile dysfunction in all patients. Impotence rates vary in the literature. Asterling et al. published a prospective evaluation of sexual function in 53 patients who underwent cryosurgery as a primary treatment for prostate cancer with 39\% of return of sexual function (28). Additionally, Lambert et al. reported up to 71\% return of potency in their study (29). Ellis et al. emphasized the application of penile rehabilitation post cryotherapy to achieve erection for intercourse with or without oral pharmacological agents. In their series, the return of potency rates was $41.5 \%$ and $51.3 \%$ of patients at 12 months and 48 months post cryo treatment respectively (30). In the only randomized trial comparing cryoablation with external beam radiation for localized prostate cancer, Donnely et al. demonstrated equivalent results between cryoablation and radiation after a follow-up of 3 years (26). Although our study had relatively few patients, $50 \%$ of these patients had erectile dysfunction before treatment. The underlying causes for this were not explored with the patients, but more men with localized prostate cancer have reported sexual problems than similarly aged men without prostate cancer in comparison group studies $(31,32)$. Some of these problems appear to be disease-linked, regardless of the type of treatment used (33). In our series, we performed early penile rehabilitation in patients that were potent pre-operatively and desired return of sexual function post-op. Interestingly, all patients that underwent post procedure penile rehabilitation were able to have sexual intercourse with penetration with pharmacological aid.

Technological and procedural modifications of cryoablation have led to a new era of cryotherapy for the treatment of prostate cancer. Indications and complications due to prostate cryoablation evolved offering a new minimally invasive modality to manage localized prostate 
cancer with minimal to no required hospital stay, acceptable quality of life and oncological outcome. Worldwide medical practices have adapted new technological advances with caution and especially nowadays they must consider adequacy of treatment including cost-effectiveness, quality of life and oncological outcomes based on scientific evidence for the treatment of localized prostate cancer.

This study has several limitations to be considered. The study is retrospective and has a very small number of patients. Oncological data still requires longer follow-up for a more robust conclusion. We did not use a validated questionnaire to access erectile dysfunction. However, this is an initial experience that demonstrates the successful treatment of localized prostate cancer by $3^{\text {rd }}$ generation cryoablation in Brazil.

\section{CONCLUSIONS}

Cryoablation is an accepted minimally invasive surgical option for the treatment of localized prostate cancer worldwide. Our initial results concur with current clinical data available since 10 patients were treated for minor complications. Third generation cryoablation has a low learning curve. Short term data seems to be promising but longer follow-up is necessary to verify oncological and functional results.

\section{CONFLICT OF INTEREST}

None declared.

\section{REFERENCES}

1. Katz AE, Rewcastle JC: The current and potential role of cryoablation as a primary therapy for localized prostate cancer. Curr Oncol Rep. 2003; 5: 231-8.

2. Cohen JK, Miller RJ: Thermal protection of urethra during cryosurgery of prostate. Cryobiology. 1994; 31: 313-6.

3. Hubosky SG, Fabrizio MD, Schellhammer PF, Barone BB, Tepera CM, Given RW: Single center experience with thirdgeneration cryosurgery for management of organ-confined prostate cancer: critical evaluation of short-term outcomes, complications, and patient quality of life. J Endourol. 2007; 21: 1521-31.
4. Baust JG, Gage AA, Robilottto AT, Baust JM: The pathophysiology of thermoablation: optimizing cryoablation. Curr Opin Urol. 2009; 19: 127-32.

5. Cohen JK, Miller RJ, Shuman BA: Urethral warming catheter for use during cryoablation of the prostate. Urology. 1995; 45: 861-4.

6. Maccini M, Sehrt D, Pompeo A, Chicoli FA, Molina WR, Kim FJ: Biophysiologic considerations in cryoablation: a practical mechanistic molecular review. Int Braz J Urol. 2011; 37: 693-6.

7. Ismail M, Ahmed S, Kastner C, Davies J: Salvage cryotherapy for recurrent prostate cancer after radiation failure: a prospective case series of the first 100 patients. BJU Int. 2007; 100: 760-4.

8. Cresswell J, Asterling S, Chaudhary M, Sheikh N, Greene D: Third-generation cryotherapy for prostate cancer in the UK: a prospective study of the early outcomes in primary and recurrent disease. BJU Int. 2006; 97: 969-74.

9. Dindo D, Demartines N, Clavien PA: Classification of surgical complications: a new proposal with evaluation in a cohort of 6336 patients and results of a survey. Ann Surg. 2004; 240: 205-13.

10. Gonder MJ, Soanes WA, Smith V: Experimental prostate cryosurgery. Invest Urol. 1964; 1:610-9.

11. Onik G, Cobb C, Cohen J, Zabkar J, Porterfield B: US characteristics of frozen prostate. Radiology. 1988; 168: 629-31.

12. Onik GM, Cohen JK, Reyes GD, Rubinsky B, Chang Z, Baust $\mathrm{J}$ : Transrectal ultrasound-guided percutaneous radical cryosurgical ablation of the prostate. Cancer. 1993; 72: 1291-9.

13. Pisters LL, von Eschenbach AC, Scott SM, Swanson DA, Dinney CP, Pettaway CA, et al.: The efficacy and complications of salvage cryotherapy of the prostate. J Urol. 1997; 157: 921-5.

14. Wong WS, Chinn DO, Chinn M, Chinn J, Tom WL, Tom WL: Cryosurgery as a treatment for prostate carcinoma: results and complications. Cancer. 1997; 79: 963-74.

15. Chin JL, Downey DB, Mulligan M, Fenster A: Three-dimensional transrectal ultrasound guided cryoablation for localized prostate cancer in nonsurgical candidates: a feasibility study and report of early results. J Urol. 1998; 159: 910-4.

16. Langenhuijsen JF, Broers EM, Vergunst $\mathrm{H}$ : Cryosurgery for prostate cancer: an update on clinical results of modern cryotechnology. Eur Urol. 2009; 55: 76-86.

17. Han KR, Cohen JK, Miller RJ, Pantuck AJ, Freitas DG, Cuevas $C A$, et al.: Treatment of organ confined prostate cancer with third generation cryosurgery: preliminary multicenter experience. J Urol. 2003; 170: 1126-30.

18. Ng CK, Moussa M, Downey DB, Chin JL: Salvage cryoablation of the prostate: followup and analysis of predictive factors for outcome. J Urol. 2007; 178: 1253-7; discussion 1257.

19. Bahn DK, Lee F, Badalament R, Kumar A, Greski J, Chernick M: Targeted cryoablation of the prostate: 7-year outcomes in the primary treatment of prostate cancer. Urology. 2002; 60: 3-11. 
20. Polascik TJ, Nosnik I, Mayes JM, Mouraviev V: Short-term cancer control after primary cryosurgical ablation for clinically localized prostate cancer using third-generation cryotechnology. Urology. 2007; 70: 117-21.

21. Dall'Oglio MF, Crippo A, Faria EF, Carvalhal GF: Sociedade Brasileira de Urologia: Diretrizes de Cancer de Prostata. Rio de Janeiro, 2011; pp. 44.

22. Mouraviev V, Nosnik I, Sun L, Robertson CN, Walther P, Albala $D$, et al.: Financial comparative analysis of minimally invasive surgery to open surgery for localized prostate cancer: a single-institution experience. Urology. 2007; 69: 311-4.

23. Jones JS, Rewcastle JC, Donnelly BJ, Lugnani FM, Pisters LL, Katz AE: Whole gland primary prostate cryoablation: initial results from the cryo on-line data registry. J Urol. 2008; 180: 554-8.

24. El Hayek OR, Alfer W Jr, Reggio E, Pompeo AC, Arap S, Srougi M: Percutaneous prostate cryoablation as treatment for high-risk prostate cancer. Clinics (Sao Paulo). 2007; 62: 109-12.

25. Cohen JK, Miller RJ Jr, Ahmed S, Lotz MJ, Baust J: Ten-year biochemical disease control for patients with prostate cancer treated with cryosurgery as primary therapy. Urology. 2008; 71: $515-8$

26. Donnelly BJ, Saliken JC, Brasher PM, Ernst SD, Rewcastle $\mathrm{JC}$, Lau $\mathrm{H}$, et al.: A randomized trial of external beam radiotherapy versus cryoablation in patients with localized prostate cancer. Cancer. 2010; 116: 323-30.

27. Long JP, Bahn D, Lee F, Shinohara K, Chinn DO, Macaluso JN Jr: Five-year retrospective, multi-institutional pooled analysis of cancer-related outcomes after cryosurgical ablation of the prostate. Urology. 2001; 57: 518-23.
28. Asterling S, Greene DR: Prospective evaluation of sexual function in patients receiving cryosurgery as a primary radical treatment for localized prostate cancer. BJU Int. 2009; 103: 788-92.

29. Lambert EH, Bolte K, Masson P, Katz AE: Focal cryosurgery: encouraging health outcomes for unifocal prostate cancer. Urology. 2007; 69: 1117-20.

30. Ellis DS, Manny TB Jr, Rewcastle JC: Cryoablation as primary treatment for localized prostate cancer followed by penile rehabilitation. Urology. 2007; 69: 306-10.

31. Helgason AR, Adolfsson J, Dickman P, Fredrikson M, Arver $S$, Steineck G: Waning sexual function--the most important disease-specific distress for patients with prostate cancer. $\mathrm{Br}$ J Cancer. 1996; 73: 1417-21.

32. Litwin MS, Hays RD, Fink A, Ganz PA, Leake B, Leach GE, et al.: Quality-of-life outcomes in men treated for localized prostate cancer. JAMA. 1995; 273: 129-35.

33. Eton DT, Lepore SJ: Prostate cancer and health-related quality of life: a review of the literature. Psychooncology. 2002; 11: 307-26.

Correspondence address: Dr. Fernando J. Kim

Chief of Urology, DHMC Director of Minimally Invasive Urological Oncology Tony Grampsas Cancer Center, UCHSC Associate Professor of Surgery, UCHSC E-mail: Fernando.Kim@dhha.org 